\title{
THE NEW MIRROR
}

Reflecting on inhabitant behaviour in VR and VR visualisations

\author{
BRANDON WANG ${ }^{1}$, TANE JACOB MOLETA ${ }^{2}$ and \\ MARC AUREL SCHNABEL ${ }^{3}$ \\ ${ }^{1}$ Victoria University of Wellington \& Aurecon \\ ${ }^{1}$ brandon.wang02@gmail.com \\ ${ }^{2,3}$ Victoria University of Wellington \\ ${ }^{2,3}$ \{Tane.Moleta|MarcAurel.Schnabel\}@vuw.ac.nz
}

\begin{abstract}
Technology inevitably evolves and develops rapidly in the modern era, industries and professions continue to strive in integrating, adapting and utilising these advancements to improve, optimise and improve the process of design to manufacture to the user experience. One such system that fits into this category is the advent of Virtual Reality and Augmented Reality. The numerous possibilities to which these visually and spatially immersive systems opportunities for immense innovation often lacks direction or an ultimate goal thus rendering this piece of software to often be little more than a visualisation tool.This paper recognises the unique position that VR allows and seeks to interrogate and deconstruct current, traditional design processes to better utilise VR in aiding and reinforcing the idea of partial testing of ideas and concepts throughout the design cycle. Different sciences such as psychology, processes and automation from computational design and considerations within software development will be employed and injected into the broader architectural context in which this research presides. In addition to the VR headset, eye tracking external hardware are integrated to develop a seamless tool and workflow that allows us, as designers to better interrogate clients behaviour within our designed digital representations which leads to validations, evaluations and criticisms of our actions within the architectural realm.
\end{abstract}

\section{Introduction}

The introduction of virtual reality environments into the architecture profession has produced a new wave of representation. However, utilization of virtual environments and the potential it offers has not often ventured out of the representation stage of design. The ability to test and track the activities within a design will have large implications for architectural design if conducted and understood, as the paper 'The Virtual Mirror: Cognitive Loads in VR and VR Visualisations' from Wang, Moleta and Schnabel begins to explore.

Intelligent \& Informed, Proceedings of the $24^{\text {th }}$ International Conference of the Association for Computer-Aided Architectural Design Research in Asia (CAADRIA) 2019, Volume 1, 535-544. (C) 2019 and published by the Association for Computer-Aided Architectural Design Research in Asia (CAADRIA), Hong Kong. 


\section{Background}

This paper is an explored continuation to the paper titled 'The Virtual Mirror: Cognitive loads in VR and VR Visualisations' by Wang, Moleta and Schnabel published in September 2018. In the previous paper, it critiqued base data such as movement and head movement that are available just by experiencing an environment, data that would allow for visual and spatial recreations of a journey which would further allow for analysis into the behavior of the inhabitants in a digital architectural space.

This paper now seeks to build upon and introduces the use of pupil data in conjunction with the last piece of research to help further understanding the mental and psychological influences of our design solutions. Single frame 'rendered' moments has traditionally been the preferred method of architectural visualisation with the advent of three dimensional, digital modelling. This method allowed for a greater control by the architect to dictate and direct the experiences of the client while providing a visually stunning yet immersively disconnected experience. However, with the introduction and development of VR headsets, the client experience has shifted to one that can be visually stunning while immersive but creates a disconnect with the architect. Relinquishing control of movement, sight and overall experience to the client.

\section{Research Question}

It is this new shift of experiential characteristics that VR allows that this body of research seeks to explore. How design perception and experiences differ between clients, designers and inhabitants by gathering and representing non-bias biometrics when a participant is presented with the digital realm and free will to experience as they see fit. The work further speculates on how the designed workflow can be used to study the behavioral characteristics, stress and cognitive loads within design processes to better inform future design decisions. By presenting the task of navigating and moving towards a destination via a digital landscape in VR; behavioral and sub-conscious characteristics caused either by the design or method of representation are found. Results will detail and recreate the participant's journey, focuses and experiences within a design.

\section{Methodology}

Due to the research topic and questions; methods regarding testing, participant requirements and recording of data will be kept consistent with the system, processing of data will also pass through the same software and scripts for interpretation and visualization. Results are to be non-bias as to ensure conclusions drawn from the tests are either concerned only with the VR system or design itself. Participants will be required to have experience with the HTC Vive to ensure the data or participant's behaviour is not influenced by one's awe of the technology or experience.

The environment is modelled and generated with Rhino 5.0 and Grasshopper which is then exported into Unity3D. The environment itself must follow the spatial restraints of the physical environment in which testing is done to ensure 
participants are moving as organically as possible. Data from Unity surrounding head direction, sight and movement will be handled via a stream writer coded within the scene that the experiment with taking place. All data will be exported and written while a participant is completing the tasks within the virtual environment, this will account for any discrepancy or system failures during or after the test.

Meanwhile, eye tracking and recording hardware from Pupil Labs will simultaneously streamwrite pupil data to a separate CSV file via the opensource software, Pupil Capture and the API developed for Unity. Both data sets are then linked into a Grasshopper script which produces data visualisation and representation in the Rhino 5.0 environment. The script will have functionalities built in that will help identify certain patterns that demonstrate a stressed state which will work in tandem with a journey slider system which will provide a visual recollection of the overall journey and points of interest. The utilisation of the tool and data provided is then dependant on the user and their interpretation. (Fig. 01)

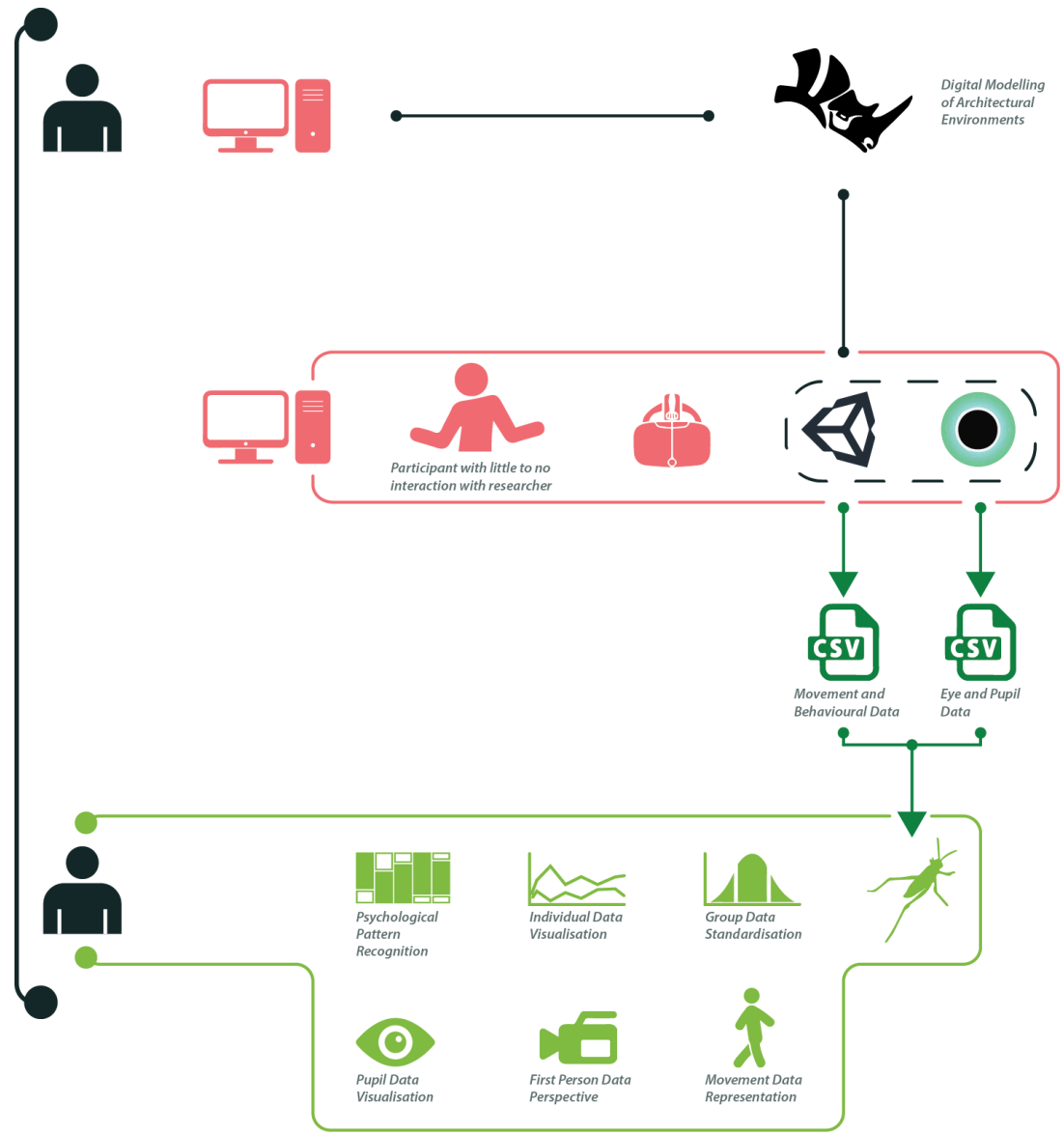

Figure 1. Author created diagram detailing work and data flow. 
Other methods of extracting data and evaluating experiences within a virtual space would be to ask participants to describe and articulate their emotions, challenges, and experiences via number sliders and self-observation. Then standardizing these resulting values to $\mathrm{Z}$ scores to place on a normal distribution to highlight problematic areas. Or, using the model of the optimal experience of Csikszentmihalyi and categorizing participants stimulation and psychological states based on the "skills" and "challenges" to determine their state of flow. However, detailed and personal data these processes provide, they are time-consuming in both the testing and processing stage and different opinions, experiences, and level of interest will influence the basic data received, potentially corrupting the results. Whereas this research is to allow for quick testing of multiple concepts and using primal reactions to validate the success of the design or inform future design decisions.

The environment chosen for the context of this research is a maze (Fig. 02). The concept of the maze is purely based around navigation. At any point, the structure allows for decisions that will either lead to a wrong path and subsequently, a dead end; or the right direction where more decisions are presented until the exit is reached. The concept of a navigational decision allows for architectural elements to be tested as a binary represented by the path that is chosen. Therefore, my design intentional as the architectural designer is to mislead and misdirect participants to dead ends using architectural elements. The number of participant's that observes these and their decisions are then a primitive result which the tool developed allows for a deeper analysis and understanding of these elements, their traits and effectiveness. The size of the maze will also be limited as participants in VR should be able to navigate and move independently without relying on hand based controls. This is to ensure the psychological and pupil data are organic as research by Zenon in 2014 details the correlation between pupil reactions and physical efforts.

\section{Design of Research}

The use of both data sets and the determination of the type of data appended are as follows:

Data from Unity is primarily aimed at visualising a recreation of the subject's journey and certain focuses throughout the architecture (Fig. 03). Body and head movement with the direction of the head is appended every 0.1 seconds, this data is then interpreted via Rhino and Grasshopper for Flow graphs, heatmaps and controlled visualisations. This base data from Unity provides sufficient data for Grasshopper to then unpack and reinterpret into points and curves which along with a user-controlled slider system, allows for a time-based walkthrough of the participant's journey from multiple perspectives. 


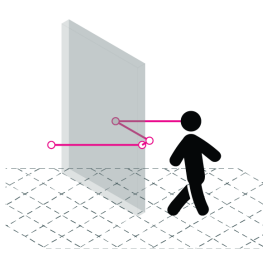

Head Movement

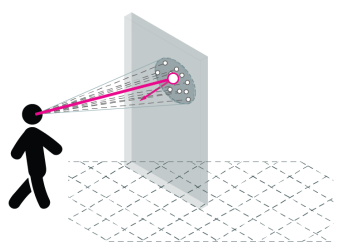

Hit Average . Hit Normal

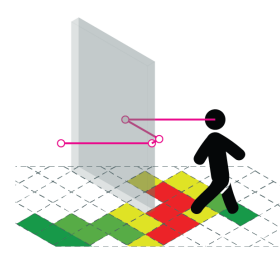

Movement Heatmap

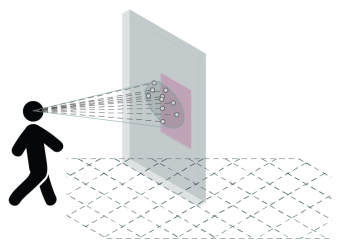

Colour Average

Figure 2. Graphic representation of base Unity3D data outputs.

The sets of pupil data from Pupil Labs include the Pupil Hippus. This "describes the oscillating pupillary unrest" (Buettner, 2014). Buettner further states that "it is known that this Hippus is strongly linked to a user's mental workload." This is captured and using base values from Buettner's research, patterns and values are coded into Grasshopper for it to begin recognising one factor of cognitive loads. Furthermore, the Pupil Diameter is a secondary method used for data validation in this research. To ensure the hardware and software are working accurately, the pupil diameters are appended alongside the Hippus and when both meet the criteria; the time is stamped and recognised as a point of interest. This method will miss areas of 'cognitive stress', however, it will verify the data integrity of the areas that are identified as both the Hippus, diameter and flow states are cross-reference for this piece of research.

\section{Final Outputs from Tool}

As stated, all data from the workflow is processed via Grasshopper and Rhino 5.0 for a final set of outputs for interpretation by the architect or designer.

- The concept of "Flow" and Flow representation. This abstraction of data was derived from both Csikszentmihalyi 's concept of Flow (Csikszentmihalyi, 1990) and Dorta's use of 'Skill' and 'Challenge' (Dorta, 2017). Within the context of this research and data, Challenge was determined by the rate of head movement while Skill was determined by the pace of movement (Fig. 04). This concept for automating the determination of flow states stem from the idea of basic navigation and sign placement and clarity. In the context of an architectural space, if one is lost within a complex structure and the behavior demonstrated includes standing with frantic head movements to find clues or signs for navigation; this is classed as a stressful moment. However, if the complexity where to be replaced with a straight hallway where the behavior demonstrated is of quick movement with minimal head activity, this is classed as a bored moment as the architecture does 
not challenge or offer any moments of challenge.

- Pupil Data. This data is primarily used for cross-referencing and validating other data sets and mentioned. The visual representation of this data offers a different method for spotting moments of interest.

- Grasshopper and slider time control. These allow for control over the moments of the journey, allowing for the architect to follow a journey step by step. The overhead view shows the position of the person within the architecture, their head and sight direction and a heatmap that demonstrates areas of movement throughout the journey (Fig. 05) . Furthermore, the plugins; Flounder and Horster for Grasshopper both allows for manipulation of cameras from Grasshopper (Fig. 06). Therefore, using the base data, head position and head movement, a first person perspective works alongside the overviews to provide a varied base for analysing and connecting the psychological and cognitive states with the architectural elements that are present.
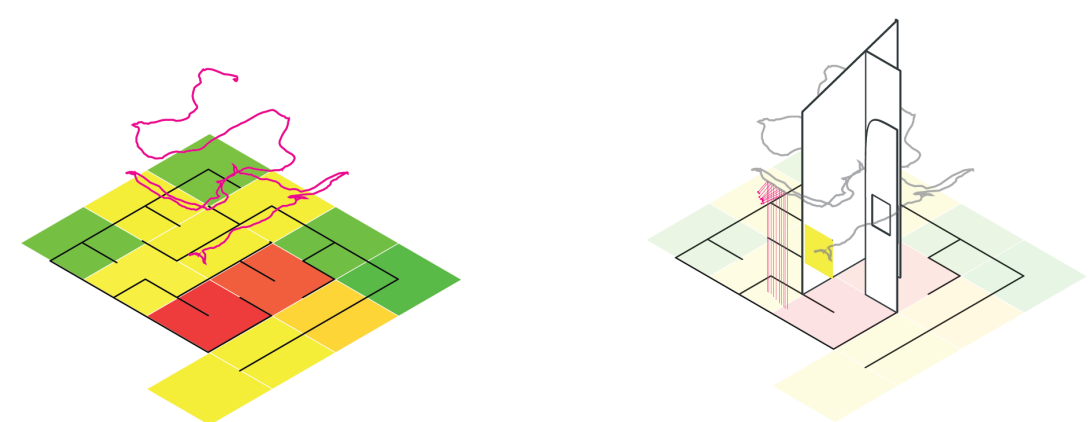

Figure 3. Overview data visualisation from Grasshopper.
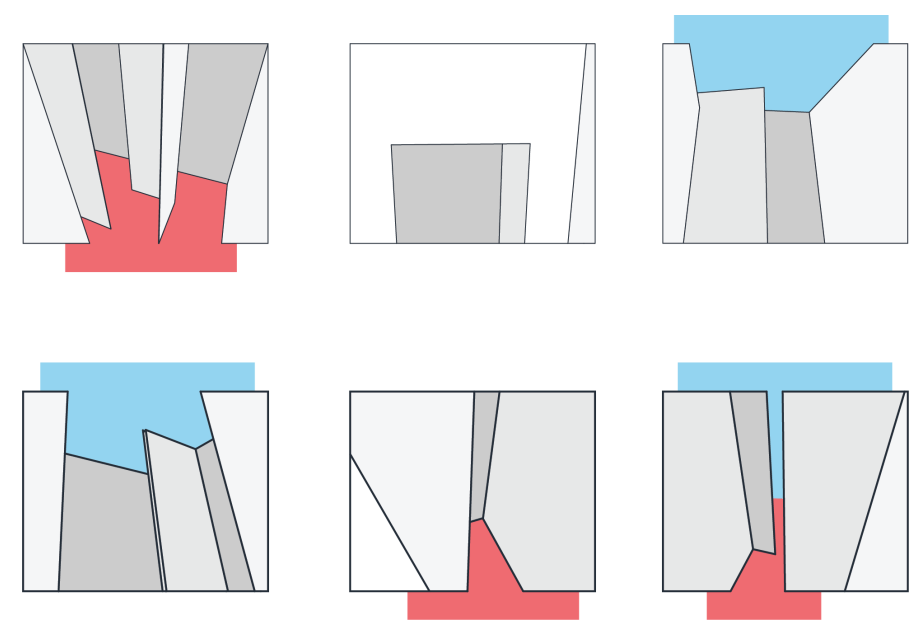

Figure 4. First person perspective snapshots from Rhino 5.0 and edited in Adobe Illustrator CC 2017. 
However, these individual sets take considerable amounts of time to analyse and process to identify recurring architectural elements or architectural languages that may or may not induce a desirable psychological effect. A basic standard deviation script made in Grasshopper takes multiple sets of Unity data and processes them to highlight general trends quickly over a larger set of data (Fig. 07). However, this model for this research has not proven effective in identifying the causes of irregular deviations as the rate, directional, focus and perception of each participant and their subsequent data is unique major events will very rarely coincide with other experiences. This is the limitation of allowing free roaming within a space therefore, another standardisation model will need to be considered as multiple individual data sets will be very difficult and time consuming to analysis individually.

\section{Initial Results}

From the initial maze, there are three 'decision' points that tests colour, geometry and wall irregularities. The results would suggest that colour, although did initially influence path taken as a majority of participant's biased to green over red. However, as participant's progress, unique coloured panels became a tool for self-orientation within the architectural maze.

Other behavioral characteristics that all participants displayed includes minimal vertical head movement. $35 \%$ of the time, lines of sight was within $0.5 \mathrm{~m}$ of the head height and $42.8 \%$ was within $0.5 \mathrm{~m}$ to $1.0 \mathrm{~m}$. Meanwhile, only $1.2 \%$ of the time, was spent looking at the floor (Fig. 08). Therefore, it would be deemed reasonable that the most influential and recognised architectural element was the window-like penetration. The placement was within the sight range that most were operating within and the offer of extra information with minimal effort piqued curiosity which then became the leading element to drive next iteration of the maze.

\section{Iterated Test}

From the preliminary tests, a main behavioural observation was that participants tend to look at eye height. Even with bare walls, therefore, the walls have been changed to a misleading chevron panel design. Higher and lower elements have been removed along with colour.

Objectives that must be completed to reach the end have also been introduced to better simulate tasks and gives a better purpose for the architecture to contain. Furthermore, the layout of the maze has been flipped to retain a sense a sense of familiarity while causing initial confusion. (Fig. 09) 

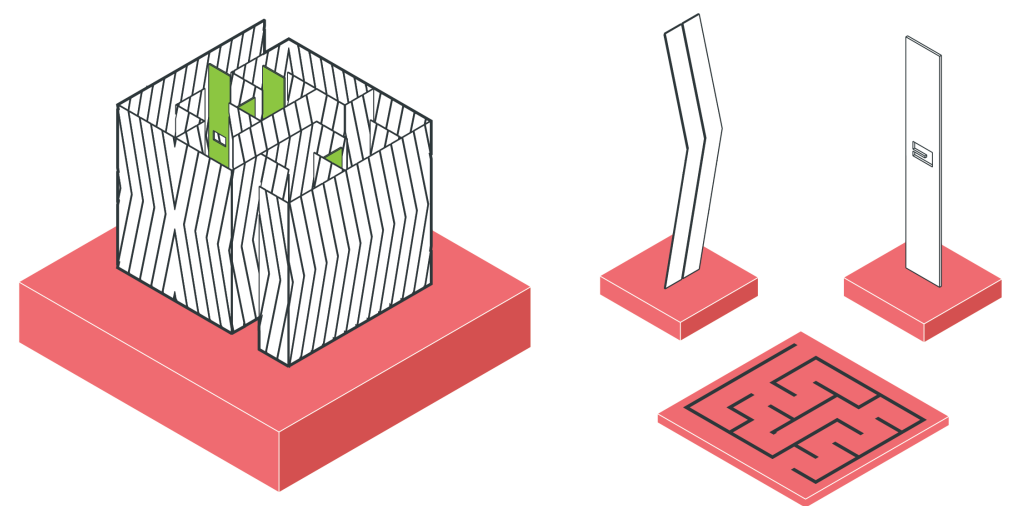

Figure 5. Iterated maze with changed architectural elements.

The introduction of the door and lock system (Fig. 10) provokes deeper thought and consideration of the space. Navigating to and locating the unique keys scattered in the maze now utilises more of the limited space. The lock and it's unique shape now also acts as subtle internal landmarks. The addition of these objectives also allows for the testing of architectural importance against personal tasks and goals.
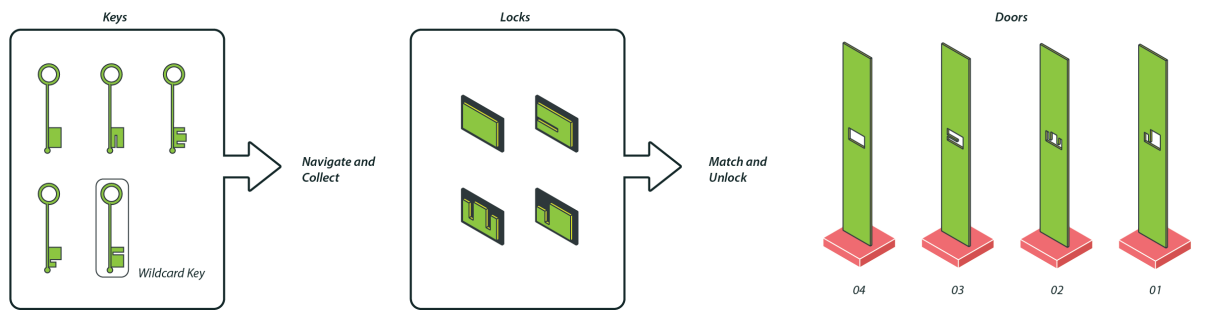

Figure 6. Graphic showing the pattern association between keys, locks and doors.

\section{Iterated Results}

Results from the same participants being run through this newest iteration of the maze showed a considerable increase in 'Flow' and overall enjoyment. This was mainly due to the fact that a challenge that the architecture facilitates was introduced. The chevron design was successful in the way that it visually disorientated participants with consistent and intensive visual complexity. However, this disorientation was only present initially and under certain circumstances within the journey. When the key and lock system was taught and understood, the architectural elements such as the chevron panelisation design was overshadowed by the task of matching the key pattern design to the lock plaques and subsequently finding the corresponding door that it opened.

With $78 \%$ of all participants ignoring the exit to the maze in favour of matching the remaining keys to the locks which were unnecessary to open, would suggest 
that the purpose of the architecture became more important and enjoyable than the initial goal of pathfinding to the end.

\section{Conclusion}

The research seeks to explore other potentials, more influential applications of virtual reality technologies. The research addresses how VAMR can be incorporated into the design process and establishes a number of confirmations in the use of VMAR for Architecture and Design. The work undertaken in this study informs how powerful a tool it could be and defines how it could be incorporated more frequently into the workflow of the conceptual and development stages of architectural design. Presently, the sandbox itself requires a level of automation that is applied throughout each test of any design and can interpret the data and help visualize it quickly. The use of pupillary response technology provides an insight into the cognitive and stress loads triggered and studying these alongside the movement and head behaviors allow for a detailed image of the potential issues and identifying the contributing elements to these issues.

This proved effective within the first iteration as the dominant areas such as the bare, white walls and attributing the type of influence from certain architectural elements were easily identified and altered to fit a design goal in the context of path finding. However, with the introduction of the internal objectives and tasks, the architectural elements were no longer the sole focus and was completely foreshadowed by the tasks at hand, only once again having an effect when participants had completely an objective and was moving to start another. The pupil data supported this as there was a notable shift in the cognitive states of when a key was matched, opening a door and when the participant was traversing the architecture; searching for the next key.

When self-evaluating this tool and research, it is clear to see the success and failures. It has been able to aid in efficiently identifying elements and directing design shifts and solutions which has created desired effects when one is inhabiting the virtual environment. However, the lack of ability to standardise large data sets and when a task is introduced such as the one within the iterated maze, clear distinctions between tasked invoked psychological states and architectural invoked psychological states can be seen but not recognised via automation. This research has provided another step into the "what do we look at?" question with further datasets and evaluations into cognitive and mental stress states. This then begs the question: "what do people do with architecture?", only then, can this tool be further developed and evolve into one that not only guides experiences but our purpose with architecture.

\section{References}

Beatty, J.B.: 1982, Task-evoked pupillary responses, processing load, Psychological Bulletin, 91, 276-292.

Buettner, R.B.: 2013, Cognitive Workload of Humans Using Artificial Intelligence Systems: Towards Objective Measurement Applying Eye-Tracking Technology, KI 2013 Proceedings, Koblenz, Germany.

Buettner, R.B.: 2014, Analyzing Mental Workload States on the Basis of the Pupillary Hippus, 
NeuroIS 2014 Proceedings: Gmunden Retreat on NeuroIS 2014, Gmunden.

Buettner, R.B., Daxenberger, B.D., Eckhardt, A.E. and Maier, C.M.: 2013, Cognitive Workload Induced by Information Systems: Introducing an Objective Way of Measuring based on Pupillary Diameter Responses, SIGHCI 2013 Proceedings.

Csikszentmihalyi, M.C.: 1990, Flow: The Psychology of Optimal Experience, Harper \& Row.

Dorta, T.D. and Marchand, E.B.M.: 2017, Immersive retrospection by video-photogrammetry UX assessment tool of interactions in museums, a case study, Proceedings of eCAADe 2017, Rome.

Innes, D.I., Moleta, T.J.M. and Schnabel, M.A.S.: 2017, Virtual Inhabitation and Creation: A Comparative Study of Interactive 1:1 Modelling as a Design Method, Conference: DADA 2017 International Conference on Digital Architecture: "Digital Culture", Nanjing.

Kvan, T.K.: 2004, The Dual Heritage of CAAD Research, International Journal of Architectural Computing, 2, 11-17.

Maver, T.M.: 1995, CAAD's Seven Deadly Sins, CAAD futures Digital Proceedings.

Sussman, A.S. and Hollander, J.B.H.: 2015, Cognitive Architecture: Designing for How We Respond to the Built Environment, Routledge, New York.

Wang, B.W., Moleta, T.M. and Schnabel, M.A.S.: 2018, The Virtual Mirror: Cognitive Loads in VR and VR Visualisations, Computing for a Better Tomorrow, 36th Annual Conference on Education and research in Computer Aided Architectural Design in Europe (eCAADe2018), Lodz, Poland.

Zenon, A.Z., Sidibe, M.S. and Olivier, E.O.: 2014, Pupil size variations correlate with physical effort perception, Frontiers in Behavioral Neuroscience. 